\title{
Manejo quirúrgico de las fracturas costales: experiencia en un centro de referencia
}

\section{Surgical management of rib fractures: experience at a referral center}

\author{
MAURICIO VelÁSqueZ, MD.(1); ViVIANA OROZCo-MARTín, MD. (2)
}

\section{Resumen}

INTRODUCCIÓN: las fracturas costales múltiples son frecuentes en el trauma torácico y se asocian con alta morbimortalidad. Su tratamiento quirúrgico ha mostrado reducir las complicaciones respiratorias, el tiempo de hospitalización, el dolor, la incapacidad funcional de los pacientes y la mortalidad.

OBJETIVO: evaluar los resultados del manejo quirúrgico de los pacientes con fracturas costales múltiples.

METODOLOGÍA: se hizo un estudio descriptivo de tipo prospectivo entre enero de 2011 y mayo de 2016, en el que se operaron 78 pacientes y se dividió la cohorte en dos grupos a fin de comparar dos periodos de tiempo en el manejo de estos pacientes e identificar si existen diferencias en los desenlaces.

RESULTADOS: existe una curva de aprendizaje no solo en el grupo quirúrgico que efectúa el procedimiento sino en todo el grupo médico que maneja los pacientes con fracturas costales múltiples.

Palabras clave: fracturas costales múltiples, trauma torácico, fijación, tórax inestable.

\begin{abstract}
INTRODUCTION: multiple rib fractures are common in chest trauma, and are associated with high morbidity and mortality rates. Surgical treatment of rib fractures has been shown to reduce respiratory complications, length of hospital stay, pain, functional disability, and mortality.

OBJECTIVE: to evaluate the results of surgical management of patients with multiple rib fractures.

METHODOLOGY: a descriptive, prospective study was carried out over the period from January 2011 to May 2016, during which 78 patients were operated. The cohort was divided in two groups, in order to compare two periods of time in the management of these patients, and thus to identify whether there were differences in the outcomes.
\end{abstract}

RESULTS: there is a learning curve, not only in the surgical group who perform the procedure, but also in the whole medical group in charge of patients with multiple rib fractures.

Keywords: multiple rib fractures, chest trauma, fixation, unstable chest wall. ${ }^{(1)}$ Cirugía de Tórax. Línea de Investigación
Biomédica en Tórax, Fundación Valle del
Lili. Facultad de Ciencias de la Salud, Uni-
versidad Icesi. Cali, Colombia.
${ }^{(2)}$ Médica Rural de Investigación. Fundación
Valle del Lili. Facultad de Ciencias de la Sa-
lud, Universidad Icesi. Cali, Colombia.
Correspondencia: Mauricio Velásquez,
correo electrónico: mauriciovelasq@yahoo.
com
Recibido: 04/09/16. Aceptado: 30/09/16. 


\section{Introducción}

Las lesiones torácicas son comunes en los pacientes politraumatizados; causan un 20 a $25 \%$ de todas las muertes traumáticas cada año en los Estados Unidos (1-3).

El tórax inestable, definido mediante radiología como tres costillas consecutivas fracturadas en dos o más segmentos y en cuanto a clínica cuando se presenta movimiento paradójico de un segmento de la pared torácica (4), se da aproximadamente en el 1 al 5\% de los pacientes con fracturas costales; sin embargo esta últimas se definen como tres o más costillas consecutivas fracturadas con desplazamiento severo y/o implosión de la pared torácica (5), son más frecuentes, en cuanto a clínica se comportan como tórax inestable y ambas entidades pueden ser responsables de una mortalidad hasta del 30 al $40 \%$ con efectos a largo plazo, como alteración en las pruebas de función pulmonar, dolor torácico crónico y sensación de disnea en un poco más de la mitad de los pacientes (1-3).

Por tradición, el manejo de los pacientes con fracturas costales múltiples había sido médico y se ingresaba al paciente a una unidad de cuidado intensivo, en tanto que el manejo quirúrgico quedaba relegado a casos muy seleccionados, entre ellos tórax inestable, incapacidad para retirar la ventilación mecánica y dolor sin respuesta al manejo médico $(2,4)$. Sin embargo, nuevos estudios demuestran que el manejo quirúrgico precoz es fundamental para una recuperación mejor y más rápida, además de la disminución de la estancia en cuidados intensivos y hospitalaria $(3,5,6)$. En tal sentido, se presenta la experiencia en el manejo quirúrgico del paciente con fracturas costales múltiples en una institución de cuarto nivel de atención en Cali, Colombia.

\section{Materiales y métodos}

Se llevó a cabo un estudio descriptivo de tipo prospectivo de los pacientes operados por tórax inestable entre enero de 2011 y mayo de 2016 en la Fundación Valle del Lili, una institución de cuarto nivel de complejidad.
Se incluyeron mayores de 18 años que tuvieran traumatismo cerrado o abierto de la pared del tórax, y dos o más fracturas costales que provocaran cualquiera de las siguientes situaciones para considerarlo como tórax inestable:

- Falla en el destete de la ventilación mecánica.

- Movimiento paradójico del tórax.

- Dolor severo o incapacitante para actividades simples como comer, orinar, toser, sentarse en el borde de la cama.

- Falla en las medidas analgésicas endovenosas, de anestesia regional o conductiva.

- Colapso de un hemitórax.

- Desplazamiento costal severo.

- Evidencia de penetrancia de las fracturas costales al tórax.

Se excluyeron aquellos que por alguna razón hubieran sido llevados a toracotomía y durante el procedimiento se les hubieran fijado las fracturas costales con cualquier método.

Se registraron variables demográficas y otras de interés como: tiempo quirúrgico, estancia hospitalaria, estancia en unidad de cuidado intensivo, lesiones asociadas, número de costillas fracturadas, necesidad y duración de la ventilación mecánica (invasiva y no invasiva), y complicaciones relacionadas con el diagnóstico y el procedimiento. La cohorte se dividió a la mitad, conformando dos grupos para comparar dos periodos en el manejo de estos pacientes e identificar si existen diferencias en los desenlaces una vez se ha ganado experiencia en el manejo de esta patología.

Para el manejo quirúrgico se utilizó una técnica quirúrgica con un abordaje planeado según el sitio de las fracturas, haciendo incisiones limitadas en la pared del tórax siempre que fuera posible y tratando de preservar la musculatura extrínseca del mismo. Se utilizó el material de osteosíntesis costal de la casa Alemana MedXpert GmbH conocido como sistema STRATOS ${ }^{\circledR}$.

Revista Colombiana de Neumología Vol 28 No 2 | 2016 


\section{Resultados}

Durante el período descrito se operaron 78 pacientes de los cuales 65 (83\%) fueron hombres y en su mayoría fueron llevados a cirugía urgente, principalmente a causa de accidentes de tránsito (tabla 1).

Se reportó una mediana de 6 costillas fracturadas: 6 en el grupo uno y 7 en el grupo dos sin diferencia entre ambos ni según el lado afectado. En cuanto a las lesiones asociadas hubo mayor presencia de fracturas del esternón, contusión pulmonar y lesiones en las extremidades en el grupo uno, así como mayor presencia de neumotórax en el grupo dos. Las demás características de las lesiones se resumen en la tabla 2.

No hubo diferencia estadística en cuanto a los días que transcurrieron entre el trauma y el momento de la cirugía, con una mediana de 2 días (tabla 3 ). Adicionalmente, hubo mayor número de pacientes con tórax inestable en el grupo uno aunque no se encontraron diferencias significativas en el número de costillas operadas ni en el de clips utilizados (tabla 3).

La estancia hospitalaria no tuvo diferencias entre los grupos con una mediana de 6 días (tabla 4). La necesidad de unidad de cuidado intensivo postoperatorio fue menor en el grupo dos aunque no alcanzó una diferencia significativa. La mediana de estancia en unidad de cuidado intensivo, así como los días de ventilación mecánica pre- y postoperatoria, no tienen diferencias estadísticamente significativas aunque hubo una tendencia a tener un día menos en la mediana de necesidad de ventilación mecánica reflejado en el grupo dos que requirió menos días de ventilación mecánica postoperatoria. Tampoco se encontraron diferencias en la morbilidad ni en la mortalidad de los pacientes operados.

\section{Discusión}

El tórax inestable es una entidad poco frecuente entre los pacientes con trauma de la pared torácica, sin embargo las fracturas costales múltiples son mucho más comunes y pueden comportarse clínicamente con una alta morbilidad, como un tórax inestable (1-5).

Hasta hace muy poco se aceptaba ampliamente que los pacientes con fracturas costales múltiples solo requerían manejo médico en cuidado intensivo y que en muy pocas ocasiones tenían indicación de

Tabla 1. Datos demográficos.

\begin{tabular}{|lrrr|}
\hline \multicolumn{1}{|c}{ Variable, $\mathbf{n}(\%)$} & $\mathbf{n}=\mathbf{5 8}$ & $\mathbf{n = 2 0}$ & p valor \\
Sexo & & & \\
Masculino & $50(86.2)$ & $15(75)$ & $0.246^{* *}$ \\
\hline $\begin{array}{l}\text { Tipo de procedimiento } \\
\text { Urgente }\end{array}$ & $40(69)$ & $14(70)$ & $0.931^{* *}$ \\
\hline $\begin{array}{l}\text { Programado } \\
\text { Remisión }\end{array}$ & $0(0)$ & & \\
Mecanismo de trauma, $\mathbf{n}(\%)$ & $18(31)$ & $6(30)$ & $0.931^{* *}$ \\
Accidente automovilístico & & & \\
\hline Caída & $35(60)$ & $9(45)$ & $0.193^{* *}$ \\
Deporte & $14(24)$ & $9(45)$ & $0.193^{* *}$ \\
Violencia & $4(7)$ & $2(10)$ & $0.193^{* *}$ \\
\hline
\end{tabular}

* Test Mann-Whitney U.

** Test Pearson-Chi . 
Tabla 2. Características del trauma.

\begin{tabular}{|c|c|c|c|}
\hline Variable & $\begin{array}{c}\text { Grupo } 1 \\
n=39\end{array}$ & $\begin{array}{c}\text { Grupo } 2 \\
n=39\end{array}$ & p valor \\
\hline \multicolumn{4}{|l|}{ Características fracturas } \\
\hline Número de costillas rotas, mediana (RIC) & $6(4-8)$ & $6(5-8)$ & 0.6460 \\
\hline Número de fracturas costales, mediana (RIC) & $6(4-9)$ & $7(5-8)$ & 0.9118 \\
\hline Derecho, n (\%) & $14(36)$ & $11(28.2)$ & 0,493 \\
\hline Izquierdo, n (\%) & $20(51.2)$ & $25(64.1)$ & 0,493 \\
\hline Bilateral, n (\%) & $5(13)$ & $3(8)$ & 0,493 \\
\hline \multicolumn{4}{|l|}{ Lesiones asociadas, $\mathbf{n}(\%)$} \\
\hline \multicolumn{4}{|l|}{ Torácicas, n (\%) } \\
\hline Esternón & $4(10.2)$ & $2(5.1)$ & 0,395 \\
\hline Clavícula & $1(2.5)$ & $5(13)$ & 0,089 \\
\hline Contusión pulmonar & $15(38.4)$ & $9(23)$ & 0,141 \\
\hline Hemotórax & $18(46.1)$ & $19(49)$ & 0,821 \\
\hline Neumotórax & $7(18)$ & $17(43.5)$ & 0,014 \\
\hline Corazón & $1(2.5)$ & $0(0)$ & 0,314 \\
\hline \multicolumn{4}{|l|}{ Abdominales, $n$ (\%) } \\
\hline Hígado & $1(2.5)$ & $2(5.1)$ & 0,556 \\
\hline Riñón & $1(2.5)$ & $1(2.5)$ & 0,986 \\
\hline Cerebro, n (\%) & $3(8)$ & $4(10.2)$ & 0,692 \\
\hline Columna, n (\%) & $0(0)$ & $2(5.1)$ & 0,152 \\
\hline Extremidades, n (\%) & $7(18)$ & $5(13)$ & 0,531 \\
\hline
\end{tabular}

manejo quirúrgico $(7,8)$. Incluso en una publicación reciente de una cohorte del National Trauma Data Bank de los Estados Unidos, de 354.945 pacientes con trauma torácico, solo 3.467 (1\%) tenían un tórax inestable, pero fue más llamativo que solo $24(0,7 \%)$ casos fueron llevados a cirugía (2). Este hecho lo explican porque los datos se recogieron durante los años 2007 a 2009, período en el que todavía no se conocían las ventajas del manejo quirúrgico, no se tenían y estaban a penas en desarrollo los materiales y dispositivos dedicados para las fracturas costales y estaban en elaboración los trabajos que han demostrado la superioridad del manejo quirúrgico frente al tradicional manejo médico.
Varios ensayos clínicos $(5,9-11)$ han demostrado que el manejo quirúrgico tiene enormes ventajas frente al médico e incluso existen metaanálisis (12, 13) que muestran disminución en los días de ventilación mecánica, en la estancia hospitalaria, en la mortalidad y en las complicaciones relacionadas con la ventilación mecánica en los pacientes llevados a cirugía.

Recientemente, nuestro grupo publicó la experiencia del manejo médico vs. quirúrgico en pacientes con fracturas costales múltiples (6) y obtuvo hallazgos similares a los descritos tanto en los ensayos clínicos como en los metaanálisis; no obstante, en vista de que esta ha sido una terapia novedosa no 
solo porque se debe tener un nuevo enfoque de manejo tanto en las unidades de trauma, como en las unidades de cuidado intensivo y los grupos de cirugía, y porque además ahora se cuenta con nuevos materiales y diseños en los dispositivos para fijar las fracturas costales, se consideró que debía existir una curva de aprendizaje y por esto se tomó la decisión de dividir la cohorte en dos grupos. Con base en los resultados no hubo diferencias significativas en ambos grupos en cuanto a las características y severidad del trauma (tabla 2), excepto por una mayor presencia de neumotórax en el grupo dos ni en cuanto a la indicación y las características del procedimiento realizado (tabla 3 ). Se debe resaltar que en el grupo dos, 4 pacientes no fueron llevados a UCI y además se encontraron tendencias (tabla 4), que si bien no logran la significancia estadística, sugieren una mejor evolución, como son un día menos de estancia en UCI ( 3 vs. 2 días) y la necesidad de ventilación mecánica postoperatoria (3 vs. 2 días), lo cual se puede explicar porque algunos pacientes fueron operados el mismo día del ingreso a la institución y el control del dolor fue adecuado, evitándose el ingreso a la UCI y pudiéndose manejar en el servicio de hospitalización general. Estos resultados sugieren que existe una curva de aprendizaje no solo en el grupo

Tabla 3. Características del procedimiento.

\begin{tabular}{|c|c|c|c|}
\hline Variable & $\begin{array}{l}\text { Menores de } 65 \text { años } \\
n=58\end{array}$ & $\begin{array}{l}\text { Mayores de } 65 \text { años } \\
\qquad n=20\end{array}$ & p valor \\
\hline $\begin{array}{l}\text { Días entre el trauma y el procedimiento, } \\
\text { mediana (RIC) }\end{array}$ & $2[1-4]$ & $2[1-4]$ & $0.821^{*}$ \\
\hline \multicolumn{4}{|l|}{ Indicación de cirugía, n (\%) } \\
\hline Tórax inestable & $14(24)$ & $8(40)$ & $0.174^{* *}$ \\
\hline Dolor agudo & $44(76)$ & $12(60)$ & $0.174^{* *}$ \\
\hline Número de costillas fijadas, mediana (RIC) & $4(4-5)$ & $5(4-6)$ & 0.056 * \\
\hline \multicolumn{4}{|l|}{ Número de clips usados, mediana (RIC) } \\
\hline Número de barras usadas, mediana (RIC) & & & \\
\hline
\end{tabular}

* Test Mann-Whitney U

** Test Pearson-Chi ${ }^{2}$

Tabla 4. Resultados.

\begin{tabular}{|c|c|c|c|}
\hline Variable & $\begin{array}{l}\text { Menores de } 65 \text { años } \\
n=58\end{array}$ & $\begin{array}{l}\text { Mayores de } 65 \text { años } \\
\qquad n=20\end{array}$ & p valor \\
\hline Estancia hospitalaria (días), mediana (RIC) & $6[5-9]$ & $6[5-12]$ & $0.662^{*}$ \\
\hline \multicolumn{4}{|l|}{ Requerimiento de $\mathrm{UCl}$} \\
\hline Estancia en UCl (días), media ( \pm ) & $2[1-5]$ & $3[2-7]$ & $0.1498^{*}$ \\
\hline Requerimiento de ventilación mecánica, n (\%) & $17(29.3)$ & $7(35)$ & $0.615^{\star *}$ \\
\hline Días de ventilación mecánica, mediana (RIC) & $5[4-14]$ & $6[3-25]$ & $0.949^{*}$ \\
\hline $\begin{array}{l}\text { Días de ventilación mecánica después de la } \\
\text { cirugía, mediana (RIC) }\end{array}$ & $3[1-4]$ & $2[1-14]$ & $0.723^{*}$ \\
\hline Morbilidad, n (\%) & $8(14)$ & $4(20)$ & $0.507^{* *}$ \\
\hline Mortalidad, n (\%) & $0(0)$ & $1(5)$ & $0.087^{\star * *}$ \\
\hline
\end{tabular}

UCI: Unidad de Cuidado Intensivo; RIC: Rango Intercuartil

* Test Mann-Whitney U

** Test Pearson-Chi ${ }^{*}$. 
quirúrgico que lleva a cabo el procedimiento sino en todo el grupo médico que maneja los pacientes con fracturas costales múltiples; como lo resaltan Pieracci et al. (5) el tiempo ha permitido refinar todo el manejo demostrando resultados comparables con la literatura mundial.

\section{Conclusiones}

Las fracturas costales múltiples son una entidad con alta morbimortalidad, en cuyo caso el manejo quirúrgico ha demostrado mejorar los desenlaces. El refinamiento del abordaje ha permitido mejorar aun más los desenlaces, hecho que se ha traducido en menores tiempos de estancia hospitalaria así como en la unidad de cuidado intensivo, además de la necesidad de ventilación mecánica con obvia disminución en las complicaciones relacionadas tanto con el traumatismo como con el procedimiento y su manejo posterior. Se requieren más estudios con un mayor número de pacientes, que permitan ajustar aún más las indicaciones precisas para el manejo quirúrgico, al igual que el mejor momento y las contraindicaciones para llevarlo a cabo.

\section{Bibliografía}

1. Kiraly L, Schreiber M. Management of the crushed chest. Crit Care Med. 2010;38:s469-77.

2. Dehghan N, de Maestral C, McKee M, Schemitsch E, Nathens A. Flail chest injuries: A review of outcomes and treatment practices from the national trauma data bank. J Trauma Acute Care Surg. 2014;76:462-8.

3. Ziegler D, Agarwal N. The morbidity and mortality of rib fractures. J Trauma Acute Care Surg. 1994;37:975-9.
4. Nirula R, Mayberry J. Rib fracture fixation: Controversies and technical challenges. Am Surg. 2010;76:793-802.

5. Pieracci F, Lin Y, Rodil M, Synder M, Herbert B, Tran DK, et al. A prospective, controlled clinical evaluation of surgical stabilization of severe rib fractures. J Trauma Acute Care Surg. 2016;80:187-94.

6. Velasquez M, Ordóñez C, Parra M, Domínguez A, Puyana J. Operative versus nonoperative management of multiple rib fractures. Am Surg. 2016(3):82:e103-5.

7. Nirula R, Diaz JJ Jr, Trunkey D, Mayberry J. Rib fracture repair: Indications, technical issues, and future directions. World J Surg. 2009;33(1):14-22.

8. Simon B, Ebert J, Bokhari F, Capella J, Emhoff T, Hayward T et al. Eastern Association for the surgery of Trauma: Management of pulmonary contusion and flail chest: an Eastern Association for the Surgery of Trauma practice management guideline. J Trauma Acute Care Surg. 2012;73(5supp14):S351-61.

9. Tanaka H, Yukioka T, Yamaguti Y, Shimizu S, Goto H, Matsuda H, et al. Surgical stabilization of internal pneumatic stabilization? A prospective randomized study of management of severe flail chest patients. J Trauma. 2002;52:727-32.

10. Granetzny A, Abd El-Aal M, Emam E, Shalaby A, Boseila A. Surgical versus conservative treatment of flail chest. Evaluation of the pulmonary status. Int Cardiovasc Thorac Surg. 2005;4:583-7.

11. Marasco S, Davies A, Cooper J, Varma D, Bennet V, Nevill $\mathrm{R}$, et al. Prospective Randomized Controlled Trial of operative rib fixation in traumatic flail chest. J Am Coll Surg. 2013;216(5):924-32.

12. Slobogean G, MacPherson C, Sun T, Pelletier M, Hameed S. Surgical fixation vs. nonoperative management of flail chest: A meta-Analysis. J Am Coll Surg. 2013;216:302-11.

13. Leinicke J, Elmore L, Freeman B, Colditz G. Operative management of rib fractures in the setting of flail chest. Ann Surg. 2013;258(6):914-21. 